\title{
On the large order behaviour of the Potts model
}

\author{
Domenico Maria Carlucci \\ Scuola Normale Superiore \\ Piazza dei Cavalieri 7 \\ 56100 Pisa, Italy
}

Internet: CARLUCCI@UX2SNS. SNS. IT

Fax number: +39 50563513

September 21, 2018

Pacs numbers: 05.20.-y, 11.10.-z, 11.10.Jj

Section proposed: General Physics 


\begin{abstract}
Following the work by Houghton, Reeve and Wallace about an alternative formulation of the $n \rightarrow 0$ limit of the $(n+1)$ state Potts model in field theory for the large order behaviour of the perturbative expansion, we generalise their technique to all $n$ by establishing an equivalence in perturbation theory order by order with another bosonic field theory. Restricting ourselves to a cubic interaction, we obtain an explicit expression (in terms of $n$ ) for the large order behaviour of the partition function.
\end{abstract}

\title{
Resumé
}

Suivant le travail de Houghton, Reeve et Wallace concernant une formulation alternative de la limite $n \rightarrow 0$ du modèle de Potts en théorie des champs pour le comportement aux grands ordres, nous généralisons leur technique à $n$ quelconque en établissant une équivalence ordre par ordre en théorie de perturbation avec une autre thórie de champs de bosons. Nous restregnant à une interaction cubique, nous obtenons une expression explicite (en fonction de $n$ ) pour le comportement aux grands ordres de la fonction de partition. 


\section{Introduction}

The Potts model [1] is one of the most fascinating topics in statistical mechanics. It can be regarded as the generalisation of the Ising model[2] to a generic number $q$ of components. Originally this problem was proposed to Potts by his PhD Professor Domb [3], who suggested him to investigate the critical properties of a system of interacting spins confined in a plane, pointing in $q$ equally spaced directions

$$
\Theta_{i}=\frac{2 \pi i}{q} \quad i=0,1, \ldots, q-1
$$

with an hamiltonian depending only on the relative orientation of two neighbouring spins of the form

$$
\mathcal{H}=-\sum_{\{\text {spins }\}} J\left(\Theta_{i}, \Theta_{j}\right) \quad J\left(\Theta_{i}, \Theta_{j}\right) \propto \cos \left(\Theta_{i}-\Theta_{j}\right)
$$

Actually, the previous model bears the name of planar Potts model. Nevertheless, the most famous model is the one where two spins interact only when they are present in the same state, that is

$$
J\left(\Theta_{i}, \Theta_{j}\right) \propto \delta_{\Theta_{i}, \Theta_{j}}^{(\mathrm{Kr})} .
$$

The interaction (3) can be re-written in such a way as to reflect a full-symmetry in a $(q-1)$-dimensional space by making use of $q$ vectors $(q-1)$-dimensional $\boldsymbol{\epsilon}^{\alpha}$ pointing in the $q$ symmetric directions of a hypertetraedron in $(q-1)$ dimensions. Indeed, the interaction (3) can be re-written as

$$
\delta\left(\Theta_{i}, \Theta_{j}\right)=\frac{1}{q}\left[1+(q-1) \boldsymbol{\epsilon}^{\Theta_{i}} \cdot \boldsymbol{\epsilon}^{\Theta_{j}}\right] .
$$

The increasing attention to the Potts model was due to the fact that it has proven to be related to a large class of outstanding problems, especially when particular values of $q$, even non integer, are taken. For example, the percolation problem can be formulated in terms of $q=1$ Potts model[4, 5, 6]. Also Fortuin and Kasteleyn[7] showed that the Kirchhoff's solution [8] for an ensemble of resistors is strictly related to $q=0$ limit of the Potts model. Moreover, the critical behaviour of the dilute spin glasses is well understood in terms of the $q=\frac{1}{2}$ limit [9, 10]. Finally, Lubensky and Isaacon [11] showed that interesting processes in gelation and vulcanization fall in the same class of universality of $0 \leq q \leq 1$ Potts model (For a complete review of the Potts model see F.Y.Wu [12]). 
A convenient formulation of Potts model as a field theory was first performed by Zia and Wallace 13, who investigated the critical properties in the framework of the renormalization group. Actually in the field theory literature it is customary to refer to $(n+1)$ Potts model $(n+1=q$ in the previous notation). The symmetry of the model allows a trilinear interaction which is the leading term in the renormalization group framework. Because of a pure cubic interaction, the high order behaviour of the perturbative expansion is clearly non-oscillating and therefore non Borel-sommable. Nevertheless, when particular limits are taken (e.g. $n \rightarrow 0$ percolation problem) the large order behaviour is found to be oscillating and Borelsommable 14) as obtained by Houghton, Reeve and Wallace (from now on referred as HRW). In Sec.I, we recall the hamiltonian of the $(n+1)$ Potts model and the alternative approach to $n=0$ limit used by Houghton, Reeve and Wallace. In Sec. II its generalisation to each value of $n$ is showed. Sec.III will be devoted to the evaluation of the large order behaviour for such an alternative field theory and the results will be shown to agree with the previous ones.

\section{The $(n+1)$-state Potts model}

We consider the $(n+1)$ state Potts model in $d$ dimension, described by an Hamiltonian 13 of the form

$$
\mathcal{H}[\boldsymbol{\phi}]=\int d^{d} x\left[\frac{1}{2}\left(\nabla \phi_{i}\right)\left(\nabla \phi_{i}\right)+\frac{1}{2} \phi_{i} \phi_{i}+\frac{g}{3 !} \rho_{i j k} \phi_{i} \phi_{j} \phi_{k}\right]
$$

where sum over repeated indices is implied and runs from 1 to $n$. The system possesses a hypertetrahedric symmetry, provided that $\rho_{i j k}$ is of the form

$$
\rho_{i j k}=\sum_{\alpha=1}^{n+1} \epsilon_{i}^{\alpha} \epsilon_{j}^{\beta} \epsilon_{k}^{\gamma}
$$

where the $\epsilon$ 's satisfy

$$
\sum_{\alpha=1}^{n+1} \epsilon_{i}^{\alpha}=0 \quad \sum_{\alpha=1}^{n+1} \epsilon_{i}^{\alpha} \epsilon_{j}^{\alpha}=(n+1) \delta_{i j} \quad \sum_{i=1}^{n+1} \epsilon_{i}^{\alpha} \epsilon_{i}^{\beta}=(n+1) \delta^{\alpha \beta}-1
$$

Physical quantities can be extracted from the partition function (the discussion about the right contour in the path integral for a pure cubic theory can be found in [15]) expanded in powers of $g$ :

$$
\mathbf{Z}_{n}(g)=\int \mathcal{D} \phi e^{-\mathcal{H}} \equiv \sum_{K=0}^{\infty} Z_{K}(n) g^{2 K}
$$


We are interested in the asymptotic behaviour of $Z_{K}(n)$ as $K$ goes to infinity. We can extract $Z_{K}(n)$ from (8) by the usual dispersion relation 16, 17]

$$
Z_{K}(n)=\int \mathcal{D} \phi \frac{1}{2 \pi i} \oint d g^{2} \frac{1}{g^{2 K}} e^{-\mathcal{H}\left[\phi_{i}\right]}
$$

where the closed integral is performed in the cut complex plane $g^{2}$. In the large $K$ limit, the previous integral can be evaluated by the steepest descent method in the space $\left(\phi_{i}, g\right)$ and the saddle point equations are

$$
\begin{gathered}
\nabla^{2} \phi_{i}=\frac{1}{2} \epsilon_{i j k} \phi_{j} \phi_{k} \\
\frac{1}{3 !} \int d^{d} x \epsilon_{i j k} \phi_{i} \phi_{j} \phi_{k}=-\frac{2 K}{g}
\end{gathered}
$$

Equations (10) and (11) can be decoupled easily by setting

$$
\Phi_{i}=\frac{1}{2} \phi_{i} \quad \Phi_{i}=u_{i} \phi_{c}(x)
$$

with the $u_{i}$ 's satisfying

$$
u_{i}=\epsilon_{i j k} u_{j} u_{k}
$$

Equation (13) has $n$ solutions $u_{i}^{(r)}$, with $r=1,2, \ldots, n$,

$$
u_{i}^{(r)}=a^{(r)} \sum_{i=1}^{r} \epsilon_{i}^{\alpha}
$$

where

$$
a^{(r)}=\frac{1}{(n+1)(n+1-2 r)}
$$

Working in $d=6$ dimension, the saddle point equations (10,11) then read

$$
\begin{gathered}
\nabla^{2} \phi_{c}(x)=\phi_{c}^{2}(x) \\
\frac{2}{3} \sum_{i} u_{i} u_{i} \int d^{6} x \phi_{c}^{3}(x)=-K g^{2}
\end{gathered}
$$

whose general solution is

$$
\phi_{c}(x)=-\frac{24 \lambda^{2}}{\left[\lambda^{2}\left(x-x_{0}\right)^{2}+1\right]^{2}}
$$

where $\lambda$ and $x_{0}$ are constant parameters. From equation (17, 18), we obtain 


$$
g^{2}=3 \pi^{3} \frac{2^{8}}{5 K} \min _{\{r\}}\left(\sum_{i} u_{i}^{(r)} u_{i}^{(r)}\right)
$$

and therefore

$$
Z_{K}(n) \sim K !\left(\frac{5}{3 \cdot 2^{8} \pi^{3} \min _{\{r\}}\left(\sum_{i} u_{i}^{(r)} u_{i}^{(r)}\right)}\right)^{K}
$$

For the $(n+1)$ state Potts model $(n>1$ integer $)$ the minimum in $(20)$ is for $r=1$, giving, as expected for a pure cubic theory, a malign behaviour for the coefficients $Z_{K}$ which do not oscillate 16, 15

$$
Z_{K}(n) \sim K !\left(\frac{5(n+1)^{2}(n-1)^{2}}{3 \cdot 2^{8} \pi^{3} n}\right)^{K}
$$

For $n=0$ (corresponding to percolation problem) the saddle point cannot be obtained by a naive analytic continuation of (20) because evidently $r=1$ is not the dominating saddle point.

HRW 14 found an alternative formulation for the percolation problem and they pointed out that in the $n \rightarrow 0$ limit the Feynman rules are the same as those of the hamiltonian with two scalar fields

$$
\begin{gathered}
\mathcal{H}(\phi, \psi, g)=\int d^{6} x\left[\frac{1}{2}(\nabla \phi)^{2}-\frac{1}{2}(\nabla \psi)^{2}+\frac{1}{2}\left(\phi^{2}-\psi^{2}\right)+\right. \\
\left.+\frac{1}{3 !} g(\phi+\psi)^{3}\right]
\end{gathered}
$$

provided that one keeps only $\phi$-connected diagrams as those shown in figure 1 . By means of path integrals techniques, they found the coefficients of the expansion of the two-point correlation function

$$
G(x, y)=\sum_{K=0}^{\infty} G_{K} g^{2 K} \quad G_{K} \sim x K !\left(-\frac{15}{2^{9} \pi^{3}}\right)^{K}
$$

which fortunately show an oscillating behaviour.

\section{HRW method for arbitrary $n$}

As mentioned in the introduction, it would be useful to generalise the previous treatment to each value of $n$. Our first task is to write down the equivalent hamiltonian in analogy with (22). In order to gain some insight, let us compute the two-point correlation function up to fourth order 


$$
\left\langle\phi_{\text {ext }} \rho_{i j k} \phi_{i} \phi_{j} \phi_{k} \rho_{l m n} \phi_{l} \phi_{m} \phi_{n} \rho_{a b c} \phi_{a} \phi_{b} \phi_{c} \rho_{d e f} \phi_{d} \phi_{e} \phi_{f} \phi_{\text {ext }}\right\rangle
$$

by performing all the possible Wick contractions. Because of the properties (7), we can get terms as

$$
\begin{gathered}
\sum_{\alpha, \beta, \gamma, \delta=1}^{n+1} \epsilon_{i}^{\alpha}\left[(n+1) \delta^{\alpha \gamma}-1\right]\left[(n+1) \delta^{\alpha \beta}-1\right]\left[(n+1) \delta^{\beta \gamma}-1\right] \times \\
\times\left[(n+1) \delta^{\beta \delta}-1\right]\left[(n+1) \delta^{\gamma \delta}-1\right] \epsilon_{f}^{\delta}
\end{gathered}
$$

Inspired by the previous discussion, we try to interpret $(n+1) \delta^{\alpha \beta}$ and $(-1)$ as propagators of two different (possibly multi-component) scalar fields, $\phi$ and $\psi$ respectively. The several terms we obtain by expanding the products in (25) correspond in the second theory to all the different diagrams with the same topology as the original ones. As in the $n \rightarrow 0$ case only diagrams with external $\phi$ legs connected by a $\phi$ 's path do contribute.

\section{** Here Figure 1 **}

In figure (1) we have drawn three of the possible graph built by $\phi$ and $\psi$ propagators. By requiring that the diagrams in the two theories have the same numerical coefficients, we obtain the Feynman rules.

Specifically, from the term involving only $\phi$ fields (figure (1a)) we get

$$
\begin{gathered}
\sum_{\alpha, \beta, \gamma, \delta=1}^{n+1} \epsilon_{i}^{\alpha}(n+1)^{5} \delta^{\alpha \gamma} \delta^{\alpha \beta} \delta^{\beta \gamma} \delta^{\beta \delta} \delta^{\gamma \delta} \epsilon_{f}^{\delta}= \\
=(n+1)^{5} \sum_{\alpha=1}^{n+1} \epsilon_{i}^{\alpha} \epsilon_{j}^{\alpha}
\end{gathered}
$$

In these case, the presence of $\delta$ 's eliminate the sum over the four indices. If we associate a factor $(n+1)$ to each $\phi$ propagator, we obtain the same results without introducing any weight for the vertices.

Let us now consider the second graph (see figure $(1 \mathrm{~b})$ )

$$
\begin{gathered}
\sum_{\alpha, \beta, \gamma, \delta}^{n+1} \epsilon_{i}^{\alpha}(n+1)^{4} \delta^{\alpha \beta} \delta^{\beta \delta} \delta^{\beta \gamma} \delta^{\alpha \beta}(-1) \epsilon_{f}^{\delta}= \\
=(-1)(n+1)^{4} \sum_{\alpha=1}^{n+1} \epsilon_{i}^{\alpha} \epsilon_{f}^{\alpha}
\end{gathered}
$$


as we said, we associated a factor $(-1)$ for the $\psi$ propagator and we do not need any factor for the vertex $\phi^{2} \psi$.

The situation is quite different when we deal with graphs where we have a $\psi^{3}$ vertex as in figure(1c) which derives from

$$
\begin{gathered}
(-1)(-1)(-1) \sum_{\alpha, \beta, \gamma, \delta=1}^{n+1} \epsilon_{i}^{\alpha}(n+1)^{2} \delta^{\alpha \beta} \delta^{\beta \delta} \epsilon_{f}^{\delta}= \\
=(-1)(n+1)^{2} \sum_{\gamma=1}^{n+1}\left(\sum_{\alpha=1}^{n+1} \epsilon_{i}^{\alpha} \epsilon_{f}^{\alpha}\right) .
\end{gathered}
$$

We note that we are left with a sum over $\gamma$ which gives a factor $(n+1)$. So a $\psi^{3}$ vertex requires a factor of $(n+1)$.

At higher order we also have non-zero diagrams with also $\phi$ connected sub-graphs as shown in figure (2)

\section{** Here Figure $2 * *$}

It can be rather simply proven that each $\phi$-connected subgraph gives a factor of $(n+1)$. Summarising the Feynman rules for $(n+1)$ component Potts model are

1) Only $\phi$-connected graphs give a non zero contribution.

2) Each $\phi$ propagator gives a factor of $(n+1)$.

3) Each $\psi$ propagator gives a factor of $(-1)$.

4) Each $\psi^{3}$ vertex gives a factor of $(n+1)$.

5) Each $\phi$-connected subgraph gives a factor of $(n+1)$.

These Feynman rules correspond to the following hamiltonian

$$
\begin{aligned}
\mathcal{H} & =\int d^{6} x\left\{\frac{1}{2} \sum_{\alpha=1}^{n+1}\left(\frac{1}{n+1}\left(\nabla \phi_{\alpha}\right)^{2}+\frac{1}{n+1} \phi_{\alpha}^{2}\right)+\right. \\
& \left.-\left(\frac{1}{2}(\nabla \psi)^{2}+\frac{1}{2} \psi^{2}\right)+\frac{g}{3 !} \sum_{\alpha=1}^{n+1}\left(\phi_{\alpha}+\psi\right)^{3}\right\}
\end{aligned}
$$

By following exactly the same procedure as HRW's in order to extract the $\phi$-connected diagrams, we get for the two-point correlation function

$$
G_{\phi-c}^{(2)}(x, y)=\frac{\int \mathcal{D} \phi \phi(x) \phi(y) \exp \left[-\mathcal{H}^{*}(\phi)\right] e^{n \mathcal{W}^{*}}}{\int \mathcal{D} \psi\left[e^{\mathcal{W}^{*}}\right]^{n+1}}+
$$




$$
\begin{gathered}
-\int \mathcal{D} \psi \mathcal{D} \phi^{(1)} \mathcal{D} \phi^{(2)} \phi^{(1)}(x) \phi^{(2)}(y) \times \\
\times \frac{\exp \left[-\mathcal{H}^{*}\left(\phi^{(1)}\right)-\mathcal{H}^{*}\left(\phi^{(2)}\right)\right]}{e^{(1-n) \mathcal{W}^{*}}\left[\int \mathcal{D} \psi e^{(n+1) \mathcal{W} *}\right]}
\end{gathered}
$$

where $\mathcal{H}^{*}(\phi)$ is the reduced hamiltonian

$$
\begin{aligned}
\mathcal{H}^{*}(\phi, \psi)=\int d x^{6}\left\{\frac{1}{2} \frac{1}{n+1}\left((\nabla \phi)^{2}+\phi^{2}\right)\right. & -\frac{1}{2} \frac{1}{n+1}\left((\nabla \psi)^{2}+\psi^{2}\right)+ \\
& \left.+\frac{g}{3 !}(\phi+\psi)^{3}\right\}
\end{aligned}
$$

and $\mathcal{W}^{*}(\psi)$ is defined by

$$
\exp \left[\mathcal{W}^{*}(\psi)\right]=\int \mathcal{D} \phi \exp -\mathcal{H}^{*}(\phi, \psi)
$$

\section{$3 \quad$ High order behaviour}

As in the $n \rightarrow 0$ case, it can be shown that only the second term on the left hand side of (30) contributes to the high order behaviour of the perturbative expansion because of the particular form of the cubic interaction and we have to evaluate

$$
\begin{gathered}
-\int \mathcal{D} \psi \mathcal{D} \phi^{(1)} \mathcal{D} \phi^{(2)} \phi^{(1)}(x) \phi^{(2)}(y) \frac{1}{2 \pi i} \oint d g^{2} \frac{1}{g^{2} K} \times \\
\times \frac{\exp \left[-\mathcal{H}^{*}\left(\phi^{(1)}\right)-\mathcal{H}^{*}\left(\phi^{(2)}\right)+2 K \log g\right]}{e^{(1-n) \mathcal{W}^{*}}\left[\int \mathcal{D} \psi e^{(n+1) \mathcal{W} *}\right]}
\end{gathered}
$$

for large $K$. Our goal can be achieved as usual by the steepest descent method. The equations for the saddle points are

$$
\begin{aligned}
& \frac{1}{n+1} \nabla^{2} \phi_{i}=-\frac{1}{2} g\left(\psi+\phi_{i}\right)^{2} \quad i=1,2 \\
& \frac{2}{n+1} \nabla^{2} \psi+\frac{1}{2} g\left(\psi+\phi_{1}\right)^{2}+\frac{1}{2} g\left(\psi+\phi_{2}\right)^{2}+(1-n) \frac{\partial \mathcal{W}^{*}}{\partial \psi}=0 \\
& \frac{1}{3 !} \int d^{6} x\left\{\left(\psi+\phi_{1}\right)^{3}+\left(\psi+\phi_{2}\right)^{3}\right\}+(1-n) \frac{\partial \mathcal{W}^{*}}{\partial g}+\frac{2 K}{g}=0
\end{aligned}
$$

It is simple to evaluate the partial derivative of $\mathcal{W}^{*}$ with respect to $g$ and $\psi$ by steepest descent method too and we obtain

$$
\frac{\partial \mathcal{W}^{*}}{\partial \psi}=\left(-\frac{1}{n+1} \nabla^{2} \psi-\frac{1}{2} g\left(\phi_{0}+\psi\right)^{2}\right)\left[1+O\left(g^{2}\right)\right]
$$


and

$$
\frac{\partial \mathcal{W}^{*}}{\partial g}=-\frac{1}{3 !}\left(\int d^{6} x\left(\psi+\phi_{0}\right)^{3}\right)\left[1+O\left(g^{2}\right)\right]
$$

provided that $\phi_{0}$ obeys to the following equation

$$
\frac{1}{n+1} \nabla^{2} \phi_{0}=\frac{1}{2} g\left(\phi_{0}+\psi\right)^{2}
$$

After the re-scaling

$$
\begin{gathered}
\phi_{i} \longrightarrow \Phi_{i}=\frac{1}{g} \phi_{i} i=0,1,2 \\
\psi \longrightarrow \Psi=\frac{1}{g} \psi
\end{gathered}
$$

the saddle point equation are finally

$$
\begin{gathered}
\frac{1}{n+1} \Phi_{i}=\frac{1}{2}\left(\Phi_{i}+\Psi\right) \quad i=0,1,2 \\
\nabla^{2} \Psi+\frac{1}{2}\left(\Phi_{1}+\Psi\right)^{2}+\frac{1}{2}\left(\Phi_{2}+\Psi\right)^{2}-\frac{(1-n)}{2}\left(\Phi_{0}+\Psi\right)^{2} \\
-2 K g^{2}=\frac{1}{3 !} \int d^{6} x\left\{\left(\Phi_{1}+\Psi\right)^{3}+\left(\Phi_{2}+\Psi\right)^{3}-(1-n)\left(\Phi_{0}+\Psi\right)^{3}\right\}
\end{gathered}
$$

In order to solve the equations (37,38, 39), let us make the ansatz

$$
\Phi_{i}=u_{i} \phi_{c}(x) \quad \Psi=\nu \phi_{c}(x)
$$

where $\phi_{c}(x)$ is the solution of (16)). Therefore, we have reduced our differential equations to algebraic ones.

$$
\begin{aligned}
& \frac{1}{(n+1)} u_{i}=\frac{1}{2}\left(\nu+u_{i}\right)^{2} \\
& \nu=-\frac{1}{(n+1)} u_{1}-\frac{1}{(n+1)} u_{2}+\frac{(1-n)}{(n+1)} u_{0} \\
& K g^{2}=\frac{3 \cdot 2^{6} \pi^{3}}{5}\left[\left(\nu+u_{1}\right)^{3}+\left(\nu+u_{2}\right)^{3}+(n-1)\left(\nu+u_{0}\right)^{3}\right]
\end{aligned}
$$

Solving the first equation gives two solution

$$
u^{( \pm)}=-\left(\nu-\frac{1}{(n+1)}\right) \pm \frac{1}{(n+1)}[1-2 \nu(n+1)]^{\frac{1}{2}}
$$

Only four choices are possible 
1) $u_{1}=u^{(+)} u_{2}=u_{0}=u^{(-)}$

2) $u_{1}=u^{(-)} u_{2}=u_{0}=u^{(+)}$

3) $u_{0}=u^{(+)} u_{1}=u_{2}=u^{(-)}$

4) $u_{0}=u^{(-)} u_{1}=u_{2}=u^{(+)}$

From the 1) and 2) we obtain two equivalent solutions provided that $n \neq 1$

$$
\nu=-\frac{2 n}{(n+1)(n-1)^{2}} \quad n \neq 1
$$

The choices 3) and 4) gives other two equivalent solution for $\nu$ when $n \neq 3$

$$
\nu=\frac{4(1-n)}{(n+1)(n-3)^{2}} \quad n \neq 3
$$

The previous two value for $\nu$ give two saddle points for $g^{2}$

$$
g_{(1)}^{2}=\frac{1}{K} \frac{3 \cdot 2^{8} \pi^{3}}{5} \frac{n}{(n+1)^{2}(n-1)^{2}} \quad n \neq 1
$$

and

$$
g_{(2)}^{2}=\frac{1}{K} \frac{3 \cdot 2^{9} \pi^{3}}{5} \frac{(n-1)}{(n+1)(n-3)^{2}} \quad n \neq 3
$$

A this point, it is interesting to note that the saddle points $g_{(1)}^{2}$ and $g_{(2)}^{2}$ are respectively the $r=1$ and the $r=2$ points of (18). Anyway, Now, our final aim is to evaluate the large order behaviour of the perturbative expansion, that is the coefficients $Z_{K}$ in the expression (8). So a little care needs to understand which saddle point dominates at $n$ fixed. We can obtain the two following behaviours

$$
Z_{K}^{(1)} \sim\left(\frac{5}{3 \cdot 2^{8} \pi^{3}} \frac{(n+1)^{2}(n-1)^{2}}{n}\right)^{K} \quad n \neq 1
$$

and

$$
Z_{K}^{(2)} \sim\left(\frac{5}{3 \cdot 2^{9} \pi^{3}} \frac{(n+1)^{2}(n-3)^{2}}{(n-1)}\right)^{K} \quad n \neq 3
$$

For $n=0$ (percolation) the second saddle point dominate over the first one and we recover the HRW results. Actually there exist other formulations for the percolation problem disagreeing with HRW [18], but it can be shown[19] that the different result was due to an unappropriate choice of an integration contour. Nevertheless, the nature of the percolation behaviour still remains controversial because there is 
another result [20] where the $r=1$ saddle point seems to dominate. For $n=1$ (Ising

model) we cannot choose the coefficient $Z_{K}^{(1)}$ and $Z_{K}^{(2)}$ is identically zero according to the fact that the trilinear interaction vanishes as $n=1$. For $n$ integer we have to take into account the (46) and this is in agreement with the calculations performed in section (四)

\section{Conclusions}

We performed the computation of the large order behaviour of the perturbative expansion of the $(n+1)$ state Potts model with a cubic interaction for a generic value of $n$, by mapping the original problem in a simpler one. We showed that our equivalent field theory has two saddle points depending on $n$ and one has to choose the smallest one in order to extract the dominant term at large order. A natural question can arise looking at the different number of the saddle points in both the formulations. Indeed in Sec.I it has been shown that the Potts model, when studied by the usual techniques, has $n$ saddle points characterised by an integer $r$, whereas our alternative formulation gives only two inequivalent saddle points. Indeed, the large order behaviour is related to the cut discontinuity of the correlation function for small value of the coupling constant, that is the imaginary part of the Green functions generated by the Taylor expansions around the saddle point. For $n=2,3, \ldots$ the imaginary part of the correlation functions is dominated by the $r=1$ saddle point. When an analytic continuation in $n$ is performed and some particular limit are taken, the $r=2$ saddle point becomes the leading term. All the other saddle points are sub-leading and they do not affect the large order behaviour. This generalisation can help to compute critical exponents for theory where $n$ is not integer as those mentioned in the introduction eventually by adding higher order interactions.

\section{Acknowledgements}

It is a great pleasure to thank Giorgio Parisi for having suggested and supervised this work and G.F.Bonini for scientific discussions.

\section{References}

[1] R. B. Potts, Proc. Camb. Phil. Soc. 48, 106 (1952). 
[2] E. Ising, Z. Phys. 21, 613 (1925).

[3] C. Domb, J. Phys A 7, 1335 (1974).

[4] P. W. Kasteleyn and C .M. Fortuin, J. Phys. Soc. Jap. (Suppl.) 26, 11 (1969).

[5] M. R. Giri, M. J. Stephen and G. S. Grest, Phys. Rev. B 16, 4971 (1977).

[6] H. Kunz and F. Y. Wu, J. Phys. C 11, L1 (1978).

[7] C. M. Fortuin and P. W. Kasteleyn, Physica 57, 536 (1972).

[8] G. Kirchhoff, Ann. Phys. Chem 72, 497 (1847)

[9] A. Aharony, J. Phys. C 11, L457 (1978).

[10] A. Aharony and P. Pfeuty, J. Phys. C 12, L125 (1979).

[11] T. C. Lubensky and J. Isaacon, Phys. Rev. Lett. 41, 829 (1978).

[12] F. Y. Wu, Rev. Mod. Phys. 54, 235 (1982).

[13] R. K. Zia and D. Wallace, J. Phys. A 81495 (1975).

[14] A. Houghton, J. S. Reeve and D. J. Wallace, Phys.Rev. B 17, 2956 (1978).

[15] A. J. McKane, Nucl. Phys. B 152166 (1979).

[16] H. Kleinert, Path Integrals in Quantum Mechanics, Statistics and OPolymer Physics, (World Scientific 1990).

[17] J. C. Le Guillou and J. Zinn Justin eds., Large Order Behaviour of Perturbation Theory, (North-Holland, 1990).

[18] A.J.McKane, J. Phys. A 19453 (1986)

[19] S.Caracciolo and D.M.Carlucci, to be published

[20] E.Brézin and A.J.McKane, J. Phys. A 202235 (1987) 


\section{List of captions}

Figure 1: Some diagrams built by means of $\psi$ (solid line) and $\psi$ (dotted line) propagators. Only the diagrams $(a)$ and $(b)$ are suitable because $\phi$ connected.

Figure 2: High order diagrams with a $\phi$ connected subgraph. The filled region means any non-zero diagram. 


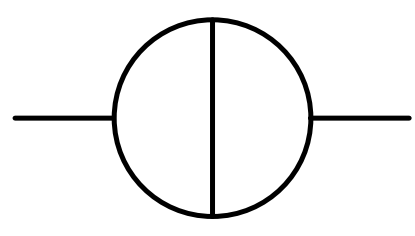

(a)

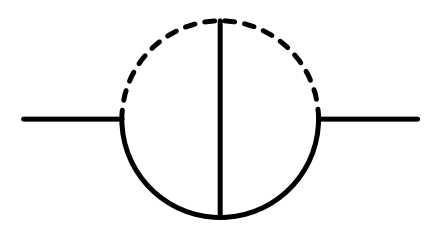

(b)

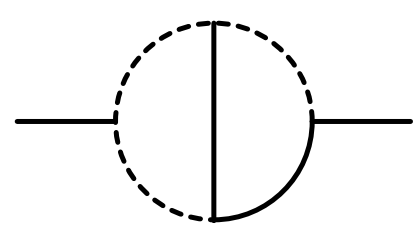

(c)

Figure 1: 


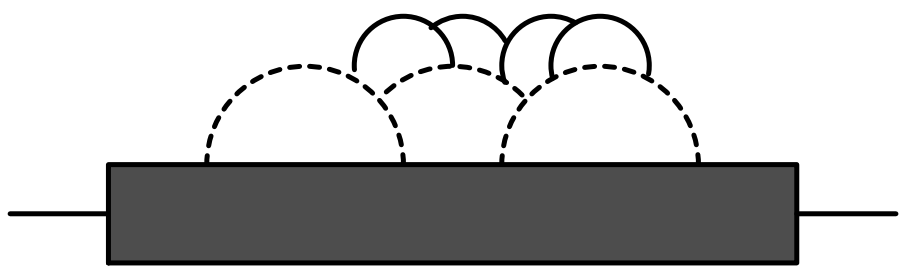

Figure 2: 